\title{
Professional discipline and support recommendations for family caregivers of persons with dementia
}

\author{
Joseph E. Gaugler ${ }^{1}$, Bonnie L. Westra ${ }^{2}$, and Robert L. Kane ${ }^{3}$ \\ ${ }^{1}$ Center on Aging, School of Nursing, University of Minnesota, Minneapolis, Minnesota, USA \\ ${ }^{2}$ School of Nursing, University of Minnesota, Minneapolis, Minnesota, USA \\ ${ }^{3}$ Center on Aging, School of Public Health, University of Minnesota, Minneapolis, Minnesota, USA
}

\begin{abstract}
Background-Family caregivers of persons with dementia often require support services to help ease the challenges of providing care. Although the efficacy of some dementia caregiver interventions seems apparent, evidence indicating which types of protocols can best meet the diverse needs of individual families is not yet available. Because of this gap, families must often turn to professionals for such guidance, but it remains unknown whether professionals from different disciplines are more inclined to recommend particular types of services than others. This study assessed whether recommendations of supportive interventions to hypothetical dementia family caregivers differed by professional discipline.
\end{abstract}

Methods-In a cross-sectional survey design, a convenience sample of 422 dementia care professionals across the USA viewed up to 24 randomly selected, hypothetical scenarios that systematically varied characteristics of persons with dementia and their caregivers. For each scenario, 7 possible intervention recommendations were rated. A total of 6,890 scenarios were rated and served as the unit of analysis.

Results-General linear models revealed that discipline was often a stronger predictor of how likely professionals were to recommend dementia caregiver interventions than caregiver, care recipient, or other professional characteristics. Psychotherapists tended to recommend psychoeducation more than other professionals, while those in medicine were more likely to recommend training of the person with dementia and psychotherapy.

Conclusions-The heterogeneity in recommendations suggests that the professional source of information influences the types of support families are directed toward. Empirical evidence should inform these professional judgments to better achieve person-centered care for families.

\section{Keywords}

Alzheimer's disease; dementia; caregiving; interventions

Correspondence should be addressed to: Joseph E. Gaugler, PhD, Center on Aging, School of Nursing, University of Minnesota, 6-153 Weaver-Densford Hall, 1331, 308 Harvard Street S.E., Minneapolis, MN 55455, USA. Phone: 612-626-2485; Fax: 612-625-7180. gaug0015@umn.edu.

Conflict of interest declaration: None. 


\section{Introduction}

Family assistance is the principal source of long-term care for persons with Alzheimer's disease throughout the world; in the USA alone over 15 million unpaid individuals (most often family members) provided 17.9 billion hours of help to 5.3 million persons with Alzheimer's disease in 2014 (The Alzheimer's Association, 2015). A well-established literature has demonstrated the adverse emotional, psychological, and health effects of dementia care on family members (Gaugler et al., 2014). As scientific evidence accumulated on the physical, financial, social, and psychological risks of dementia family care, a series of intervention strategies were developed and evaluated. The premise of these approaches was to test whether certain aspects of dementia caregiving were modifiable in order to improve key outcomes (e.g. enhancing caregiver strategies to manage dementia-related symptoms; bolstering resources through enhanced social support; Zarit, 2009). Multiple meta-analyses and systematic reviews of these interventions revealed that dementia caregiver interventions that are delivered over time, tailored to meet the individual needs of caregivers and care recipients, and include multiple components are most effective (Pinquart and Sörensen, 2006; Gitlin and Hodgson, 2015). These meta-analyses tend to include studies that: (a) use a range of controlled designs (e.g. quasi-experimental in addition to randomized controlled trial designs); (b) rely on smaller sample sizes that may increase the risk of Type II error; and (c) are often grouped or classified in inconsistent ways, among other limitations. For these reasons, the quality of available evidence remains in question. Nonetheless, several of these protocols are now implemented as part of routine clinical care in various community organizations and healthcare settings throughout the USA (Nichols et al., 2014; Wethington and Burgio, 2015).

Although the efficacy of some dementia caregiver interventions is apparent, it remains unclear which types of protocols can best meet the diverse needs of individual families. Specifically, there remain few empirically-derived, individualized information tools or aides that can help caregivers themselves determine whether a particular intervention approach is "right" for them or the person with dementia (Anderson et al., 2009; Gaugler et al., 2014). Due in part to this lack of well-organized, self-accessible information, caregivers may turn to professionals for advice.

Members of different professions may be more inclined to recommend particular types of services than others. Ideally, such recommendations would be informed by a strong evidence base that would match the needs and preferences of the caregiver and the cognitively impaired care recipient with an appropriate intervention, but such data are lacking. In an analysis of professional recommendations of long-term care services for older adults, physicians, and nurse practitioners were more likely to recommend nursing homes as a longterm care option to older adults with various care needs than other professionals, even with the significant policy and service delivery efforts in the USA to "rebalance" long-term care away from nursing homes and towards community-based long-term supports (Kane et al., 2006). Nurses and physicians appeared to base their recommendations primarily on the services with which they were most familiar. It remains unknown whether a similar mix of clinical experience and disciplinary biases influence professionals who assist dementia caregiving families. 
Interprofessional collaboration, education, and service models are essential to optimal dementia care (Gould and Reed, 2009; Rampatige et al., 2009; Brody and Galvin, 2013), but extensive variation in professional recommendations for dementia caregiver support services may complicate how such models perform. In addition, inconsistent advice may hinder families' searches for intervention approaches that are appropriate for their particular needs. Identifying disciplinary variations in dementia caregiver support recommendations would not only reveal the extent of such inconsistencies, but may also suggest new insights for how professionals are trained and certified to work with families. For example, highlighting disciplinary variations may help to emphasize the need for more standardized training to better guide professionals when making recommendations to families.

The present study asked a diverse sample of dementia care professionals across the USA to recommend support strategies for hypothetical dementia caregivers. Each hypothetical scenario focused on family caregivers of persons with dementia living in a community setting (e.g. home, independent living, non-nursing home residence). The objective was to assess whether significant differences in recommendations to dementia family caregivers were associated with professional discipline, and whether such associations were stronger when compared to other professional, caregiver, and care recipient indicators.

\section{Methods}

\section{Sample}

A convenience sample of professionals from a wide range of disciplines was recruited. Professionals who had scholarly expertise in dementia family caregiving and/or provided direct services to a family caregiver of someone with memory loss were targeted. Specifically, email lists were created from publicly available directories of family caregiver support specialists, directors, and related administrative staff in all area agencies on aging throughout the USA in the summer of 2013. In addition, emails lists of organizations that represent geriatric social workers, gero-psychologists, geriatricians, nurse care managers, geriatric nurse practitioners, and staff in these organizations (e.g. the American Geriatrics Society, the Family Caregiver Alliance, multiple LinkedIn groups) were created. The organizations identified included public service programs and private foundations and associations. Email lists of individual research and professional experts in dementia caregiving (based on the first author's experience in the field and his professional network) were also assembled. A list of $n=3,177$ emails was created using these procedures. Email invitations to participate in the project were then distributed. Respondents did not receive any incentive or compensation for completing the survey. These efforts resulted in a sample of 422 professional raters. This broad-based recruitment strategy helped to enroll a professional sample that varied in terms of discipline, geographic location, length of employment, and care sector (see Table 1).

\section{Procedure}

The study procedure was similar to that of Kane and colleagues (Kane et al., 2006). Hypothetical scenarios of dementia family caregiver situations were created and characteristics of caregivers and persons with dementia were randomly varied. A validated 
risk screen, the Risk Appraisal Measure (RAM), was used to describe the specific characteristics of the hypothetical caregiver and person with dementia for each care scenario. The RAM is a brief, validated 16-item assessment tool that includes six dimensions associated with negative health outcomes for the person with dementia or the caregiver (Czaja et al., 2009): whether the caregiver exhibits/indicates depressive symptoms, burden, poor self-care, and lack of social support and whether the care recipient exhibits behavior problems and safety problems. In addition to the six dimensions of the RAM, four additional contextual variables were included based on their empirical associations with dementia caregiver outcomes and persons' with dementia residential long-term care admission (diagnosis/dementia type, kin relationship of caregiver to the person with dementia, whether the caregiver lives with the person with dementia, and dementia stage; see Table 2) (Gaugler et al., 2009). Raters viewed a series of dementia caregiver scenarios in which the six caregiver and person with dementia RAM domains in the left-hand column of Table 2 were presented. Each professional repeated this rating task on subsequent scenarios where the four contextual characteristics (the 2nd column in Table 2) were modified one at a time in each scenario.

Three randomly selected combinations of the six RAM domains (i.e. three scenario "sets") were distributed to participating dementia care professionals. Each scenario set included eight individual scenarios. An example scenario set appears in Figure 1. The 1st paragraph of text in Figure 1 represents the six RAM dimensions that were fixed for that scenario set (and were randomly varied for two other scenario sets provided to each professional). Characteristics in the 2nd paragraph (the right hand column of Table 2) were then varied one at a time to construct each hypothetical dementia caregiver scenario. There were 504 scenarios possible using this approach.

Seven potential intervention recommendations were provided to dementia care professionals, who were asked to determine which intervention type was most appropriate for each individual scenario. Intervention types were based on prior meta-analyses of dementia caregiver interventions; they included psychoeducation, case management/ counseling, support groups, respite, training of the person with dementia (cognitive rehabilitation), psycho-therapy, and multi-component approaches (e.g. combinations of multiple service modalities, such as individual and family counseling as well as support groups). These intervention types broadly align with previous classifications of dementia caregiver interventions (Pinquart and Sörensen, 2006; Gitlin and Hodgson, 2015).

Dementia care professionals rated each intervention/support strategy's appropriateness for each scenario on a scale of 1 to 7 , with 1 as the highest recommendation and 7 as the lowest recommendation (these scores were reversed for ease of interpretation in subsequent analyses). When rating scenarios professionals were not asked to target a specific outcome but instead were advised to recommend the particular intervention that best suited the hypothetical caregiver. Scenarios were based on the assumption that they represented family caregivers of persons living in a non-institutional setting (e.g. home, independent living).

Scenario recommendations were collected via online surveys which included directions on how to complete recommendations for each scenario as well as descriptions of each 
dementia caregiver intervention type. In addition to scenario ratings, respondents were asked to provide basic background information (e.g. professional discipline, gender, highest educational degree, race, ethnicity).

\section{Analysis}

General linear models were conducted to examine whether professional discipline was significantly associated $(p<0.05)$ with dementia caregiver intervention recommendations for each scenario. Individual scenarios $(N=6,890)$ were the unit of analysis. Given the possible number of item combinations for the dementia caregiver scenario elements in Table 2 , not all scenario combinations were created for professionals to rate. However, the statistical approach and design selected allowed us to adjust for caregiver and person with dementia risk factors (i.e. the variables included in Table 2) as well as other professional background characteristics (see Table 1). Specifically, each model examined whether care discipline was statistically associated with strength of recommendation for each intervention after adjusting for variations in caregiver, person with dementia, and other professional characteristics. Predictors and covariates were standardized to allow for comparison of regression coefficients. IBM SPSS version 21 was utilized to conduct all analyses (IBM Corporation, 2012).

\section{Results}

Table 2 provides background information on professional discipline, care sector, gender, race/ethnicity, years of experience, and geographic location of clientele for raters who completed scenario recommendations. As Table 2 suggests, a diverse set of 422 dementia care professionals provided intervention recommendations for at least one scenario. A total of 6,890 individual scenarios were rated. Professionals rated an average of 21 scenarios (SD $=6.75$, Range $=1-24)$.

The separate disciplinary and sector classifications presented in Table 2 were combined for subsequent analyses due to small numbers of respondents in some categories. Combined categories included the following disciplines: Social work; Nursing (Advanced Practice Nurse/APN, Certified Nurse Specialist/CNS, Registered Nurse/RN, Licensed Practical Nurse/LPN); Medicine (Geriatrics, Family Practice, Primary Care, Psychiatry); Rehabilitation (Occupational Therapy/Physical Therapy); Psychotherapy (Psychology/ Marriage \& Family Therapy); Administration (Public Health/Long-Term Care Administration/Social Services Administration); Gerontology; and Other (all remaining disciplines). Several professionals entered care disciplines that did not align with the fixed choice options provided on the surveys: these included "dementia expert," "coach," "advocate," or similar responses. As these disciplines could not be integrated into the existing categories and the number of individuals in each was too small to assign them their own individual classification, an "Other" discipline category was created. The separate sectors were also combined into the following categories: Hospital; Non-Profit Organization; Community Service Provider; Residential Long-Term Care; Home Care/Personal Services/ Home HealthCare; and Other (all remaining care sectors that could not be categorized). 
Average strength of recommendation for each type of caregiver intervention on the 7-point recommendation scale was as follows: case management and counseling $=4.98(\mathrm{SD}=1.70)$; psychoeducation $=4.79(\mathrm{SD}=1.79)$; support groups $=4.51(\mathrm{SD}=1.69) ;$ multicomponent interventions $=4.39(\mathrm{SD}=2.22)$; respite $=4.18(\mathrm{SD}=1.93)$; psychotherapy $=3.39(\mathrm{SD}=$ 2.02); and cognitive rehabilitation/training of the person with dementia $=3.11(\mathrm{SD}=2.13)$. Multi-component interventions (29.5\%), case management/counseling (24.1\%), and psychoeducation (22.1\%) received the highest recommendation (7) most frequently. In contrast, $15.4 \%$ of support group ratings, $13.9 \%$ of respite, $11.3 \%$ of psychotherapy, and $9.7 \%$ of training of the person with dementia ratings were given the highest recommendation.

Statistically significant associations between professional characteristics and dementia caregiver intervention ratings are displayed in Table 3 adjusting for variations in caregiver (depression, burden, at-risk for poor self-care, lacks social support, lives with care recipient, kin relationship, with care recipient), person with dementia (behavior problems, at-risk for safety problems, Alzheimer's disease diagnosis, dementia stage), and professional characteristics (race, ethnicity, gender, years of professional experience, care sector, geographic location of clientele) in each scenario. Disciplinary and care sector comparisons were made against their respective other reference categories.

A review of the standardized regression coefficients in Table 3 suggests that professional discipline was usually stronger than other covariates when accounting for caregiver intervention recommendations. For example, psychotherapists recommended psychoeducation to dementia caregivers more highly $(\beta=0.36, p<0.001)$, whereas those in medicine and public health/administration were less likely to recommend case management and counseling $(\beta=-0.27, p<0.001 ; \beta=-0.25 ; p<0.001$, respectively). Other aspects of professionals' backgrounds were more strongly related to case management and counseling recommendations; for example, professionals in hospitals or community service settings were not as enthusiastic when suggesting case management and counseling $(\beta=-0.32, p<$ $0.001 ; \beta=-0.39, p<0.001$; respectively).

Professional discipline was most strongly related to support group recommendations. Occupational and physical therapists and nurses were less likely to endorse support groups for hypothetical caregivers $(\beta=-0.45, p<0.001 ; \beta=-0.32, p<0.001$, respectively). Gerontologists were also less enthusiastic in suggesting support groups $(\beta=-0.39, p<$ $0.001)$. Professionals in medicine were more likely to recommend respite highly $(\beta=0.24, p$ $<0.001$ ), although other factors appeared more strongly related to such recommendations. Professionals who had 15-25 years of work experience were less likely to endorse respite when compared to those with over 25 years of experience $(\beta=-0.28, p<0.001)$. Professionals in hospital or home care settings were less enthusiastic when recommending respite $(\beta=-0.29, p<0.001 ; \beta=-0.28, p<0.001$, respectively). Professionals were more enthusiastic when supporting respite if caregivers lived with care recipients in the hypothetical scenarios $(\beta=0.25, p<0.001)$.

Care discipline and care sector were most strongly associated with recommendations related to training the person with dementia. Professionals in medicine were more likely to support 
training for the person with dementia $(\beta=0.52, p<0.001)$ as were professionals in home care settings $(\beta=0.52, p<0.001)$. Similarly, care discipline was strongly linked to psychotherapy recommendations. Professionals in medicine were more enthusiastic when recommending psychotherapy $(\beta=0.51, p<0.001)$. The most prominent predictor of multicomponent recommendations was care sector. Professionals in the home care sector were less likely to recommend multi-component interventions to dementia caregivers $(\beta=-0.39$, $p<0.001)$, while those in hospital setting were more likely to do so $(\beta=0.35, p<0.001)$.

\section{Discussion}

The main finding of this study is that professional discipline consistently influenced intervention recommendations for hypothetical dementia caregivers, and that such differences were significant even after adjusting for other important professional characteristics and caregiver or care recipient risk factors. For example, professionals of multiple disciplines tended to have less enthusiasm for certain types of interventions. This pattern of findings emerged most strongly for support groups. Such results suggest a partial alignment between practice and evidence for some professional disciplines. Although promising results for targeted, highly structured support groups led by trained professionals are evident (Thompson et al., 2007), meta-analyses suggest moderate to no statistically significant effects of support group participation on dementia caregiver outcomes (Pinquart and Sörensen, 2006; Gitlin and Hodgson, 2015). Similarly, psychotherapists and medical professionals may have viewed dementia caregiving in the context of more global chronic disease models of clinical practice, and thus recommended more comprehensive and intensive service options such as psychoeducation and psychotherapy. A range of protocols that aim to provide caregivers with skills to effectively manage dementia symptoms have demonstrated positive effects across multiple dementia caregiver outcomes (Gaugler et al., 2014; Gitlin and Hodgson, 2015; The Alzheimer's Association, 2015).

In contrast, most other professional disciplines' recommendations were inconsistent with scientific evidence. For example, medical professionals tended to demonstrate more enthusiasm for respite. Respite is generally popular and viewed as a frontline service for family caregivers of persons with dementia (Anderson et al., 2013; Doty and Spillman, 2015), given the efforts to "rebalance" long-term care towards home- and community-based services in the USA and in other countries. However, respite has failed to demonstrate consistent effects on dementia caregivers or persons with dementia in high quality, randomized controlled evaluations (Schoenmakers et al., 2010; Jones et al., 2012). Although multi-component interventions are often suggested as among the most effective dementia caregiver interventions (Burgio et al., 2016; Gitlin and Hodgson, 2015), professionals across disciplines did not demonstrate robust enthusiasm for these approaches. As the findings of this study suggest, it is likely that professionals tended to offer recommendations that were more commonly accepted in their spheres of practice, instead of recommending interventions that have demonstrated efficacy for dementia caregivers (see Kane et al., 2006).

There are several limitations of this study. The convenience sample is not nationally or internationally representative of professionals who serve dementia caregivers. Because of the 
wide range of methods utilized to invite various individuals to participate (in addition to some professionals distributing their invitations to others); a true response rate was not available. Due to a small number of professionals in certain categories (e.g. those with different nurse degrees) some disciplines were combined; this step may have obscured disciplinary differences. As noted earlier, it was not possible for experts to rate all possible combinations of answers on the dementia caregiver scenario elements in Table 2. This limitation was partially mitigated by holding caregiver, care recipient, and professional characteristics constant, thus controlling for their effects in the analysis. In addition, there is no consistent categorization of dementia caregiver intervention types (Gitlin and Hodgson, 2015). Our labeling of interventions may have excluded other important modalities. Although disciplinary differences emerged in our analysis, we do not have in-depth data available as to why these variations occurred, or whether participants perceived the heterogeneous characteristics present in each scenario in different ways.

A principal reason families must rely on professional recommendations is that the current evidence base does not provide clear answers as to whether certain interventions are more effective than others for different types of dementia caregivers (Van Mierlo et al., 2012). In a recent evidence-based synthesis of over 30 existing systematic reviews and meta-analyses of dementia caregiver interventions, Gitlin and Hodgson (2015) found that multi-component interventions appeared most efficacious in reducing caregiver burden and delaying care recipient institutionalization, and education-focused interventions were most effective in improving caregiver knowledge. Relaxation-based interventions had the largest effects on reducing caregiver anxiety while psychoeducational interventions appeared most likely to alleviate caregiver depression. Overall, effect sizes across outcomes were moderate or small, and it remains unclear whether certain interventions vary in their effects across caregivers of different kin relation, living arrangement, and care recipient dementia severity. The current study serves to further highlight this scientific and clinical gap as it finds that disciplinary background dictated clinicians' recommendations.

The heterogeneity in training and experience that influences variability in dementia caregiver support recommendations underscores the need for improved training of professionals to help guide the selection of interventions for different types of dementia caregivers. Certification could also help professionals build on their clinical experience and extend beyond the bounds of professional discipline or care sector. Results such as those presented here point to the necessity of developing training that involves the knowledge and skills required to work with families caring for relatives with dementia. Such conclusions align with the goals, recommendations, and objectives of the National Alzheimer's Plan in the USA (http://aspe.hhs.gov/daltcp/napa/NatlPlan2014.shtml) as well as national dementia strategies in Europe and elsewhere (see Downs et al., 2009; http://www.alzheimereurope.org/Policy-in-Practice2/National-Dementia-Plans). In particular, our results are consistent with the recommendations of the United States' National Alzheimer's Plan's Strategy 2.A: To Build a Workforce with the Skills to Provide High-Quality Care (i.e. "These providers need accurate information about caring for someone with Alzheimer's disease including the benefits of early diagnosis, how to address the physical, cognitive, emotional, and behavioral symptoms of the disease, and how to assist caregivers as they cope with the physical and emotional aspects of their caregiving responsibilities"). Recent 
efforts to help achieve these goals include grants to Geriatric Education Centers through the Health Resources and Services Administration to educate healthcare providers such as physicians, social workers, nurses, and certified nursing assistants to help address caregivers' needs. However, it remains unclear if these efforts will result in a formal process that certifies core sets of skills and education required to work with dementia family caregivers. If the results here are any indication, such training will have to overcome tendencies to recommend services according to disciplinary conventions.

The merging of clinical opinion with empirically-derived recommendations based on comparative trials or similar methods (Wethington and Burgio, 2015) would greatly advance how such services are delivered to and used by families caring for relatives with dementia. The successful integration of these two sources of information could help to generate individualized service recommendations for dementia caregivers by: (1) identifying the recommended dementia caregiver intervention/support service that can best achieve the desired outcome (reduced caregiver distress; delayed nursing home admission of the person with dementia) via an individualized assessment of need; and (2) providing the necessary information to a dementia family caregiver to facilitate the selection of a recommended support strategy (Kane et al., 2006). As scientific evidence of dementia caregiver efficacy continues to accumulate, efforts to incorporate this information into clinical tools that help families identify the intervention that is most appropriate for their situation will allow evidence-based protocols to reach more dementia family caregivers in need.

\section{Acknowledgments}

The authors would like to thank Dr. Julie Bershadsky for her analytic assistance on this manuscript. Funding sources and related paper presentations: The research reported here was supported by grants R03 HS020948 (Agency for Healthcare Research \& Quality) and K18 HS022445 (Agency for Healthcare Research \& Quality) to Dr. Gaugler. A version of this paper was presented as a poster by the authors at the 2015 Gerontological Society of America conference in Orlando, Florida, USA.

Description of authors' roles: J. E. Gaugler was responsible for all aspects of the study, including the design (i.e. recruitment, enrollment, data collection, and data management), analysis, and the writing of this manuscript. B. L. Westra was Co-Investigator on the grant that principally supported this work; she offered technical guidance in the development of the survey and also reviewed and revised the current manuscript. R. L. Kane was the architect of the study procedure that this project is based upon, is Co-Investigator on the primary grant that funded this work, and was responsible for its conceptualization and analytic approach. He also reviewed and revised the manuscript.

\section{References}

Anderson KA, Dabelko-Schoeny H, Johnson TD. The state of adult day services: findings and implications from the MetLife national study of adult day services. Journal of Applied Gerontology. 2013; 32:729-748. [PubMed: 25474796]

Anderson KA, Nikzad-Terhune KA, Gaugler JE. A systematic evaluation of online resources for dementia caregivers. Journal of Consumer Health on the Internet. 2009; 13:1-13.

Brody AA, Galvin JE. A review of interprofessional dissemination and education interventions for recognizing and managing dementia. Gerontology \& Geriatrics Education. 2013; 34:225-256. DOI: 10.1080/02701960.2013.801342 [PubMed: 23879387]

Burgio, LD., Gaugler, JE., Hilgeman, M. Family Caregiving for Adults and Elders with Chronic Conditions. New York, NY: Oxford University Press; 2016.

Czaja SJ, et al. Development of the risk appraisal measure: a brief screen to identify risk areas and guide interventions for dementia caregivers. Journal of the American Geriatrics Society. 2009; 57:1064-1072. DOI: 10.1111/j.1532-5415.2009.02260.x [PubMed: 19453305] 
Doty, P., Spillman, B. Help for family caregivers available from government programs and policies. In: Gaugler, JE., Kane, RL., editors. Family Caregiving in the New Normal. San Diego, CA: Elsevier, Inc; 2015. p. 153-190.

Downs M, Capstick A, Baldwin PC, Surr C, Bruce E. The role of higher education in transforming the quality of dementia care: dementia studies at the University of Bradford. International Psychogeriatrics. 2009; 21(Suppl. 1):S3-S15. DOI: 10.1017/S1041610209008837 [PubMed: 19317922]

Gaugler JE, Potter T, Pruinelli L. Partnering with caregivers. Clinics in Geriatric Medicine. 2014; 30:493-515. doi:S0749-0690(14)00038-X[pii]. [PubMed: 25037292]

Gaugler JE, Yu F, Krichbaum K, Wyman JF. Predictors of nursing home admission for persons with dementia. Medical Care. 2009; 47:191-198. DOI: 10.1097/MLR.0b013e31818457ce [PubMed: 19169120]

Gitlin, LN., Hodgson, N. Caregivers as therapeutic agents in dementia care: the evidence-base for interventions supporting their role. In: Gaugler, JE., Kane, RL., editors. Family Caregiving in the New Normal. San Diego, CA: Elsevier, Inc; 2015. p. 305-356.

Gould E, Reed P. Alzheimer's association quality care campaign and professional training initiatives: improving hands-on care for people with dementia in the USA. International Psychogeriatrics. 2009; 21(Suppl. 1):S25-S33. DOI: 10.1017/S1041610209008758 [PubMed: 19288968]

IBM Corporation. IBM SPSS Statistics for Windows. Armonk, NY: IBM Corporation; 2012.

Jones C, Edwards RT, Hounsome B. A systematic review of the cost-effectiveness of interventions for supporting informal caregivers of people with dementia residing in the community. International Psychogeriatrics. 2012; 24:6-18. DOI: 10.1017/S1041610211001207 [PubMed: 21813034]

Kane RL, Bershadsky B, Bershadsky J. Who recommends long-term care matters. The Gerontologist. 2006; 46:474-482. [PubMed: 16921001]

Nichols LO, Martindale-Adams J, Burns R, Zuber J, Graney MJ. REACH VA: moving from translation to system implementation. The Gerontologist. 2014 doi:gnu112[pii].

Pinquart M, Sörensen S. Helping caregivers of persons with dementia: which interventions work and how large are their effects? International Psychogeriatrics. 2006; 18:577-595. [PubMed: 16686964]

Rampatige R, Dunt D, Doyle C, Day S, van Dort P. The effect of continuing professional education on health care outcomes: lessons for dementia care. International Psychogeriatrics. 2009; 21(Suppl. 1):S34-S43. DOI: 10.1017/S1041610209008746 [PubMed: 19288970]

Schoenmakers B, Buntinx F, DeLepeleire J. Supporting the dementia family caregiver: the effect of home care intervention on general well-being. Aging \& Mental Health. 2010; 14:44-56. [PubMed: 20155520]

The Alzheimer's Association. 2015 Alzheimer's disease facts and figures. Alzheimer's \& Dementia. 2015; 11:332-384. DOI: 10.1016/j.jalz.2015.02.003

Thompson CA, Spilsbury K, Hall J, Birks Y, Barnes C, Adamson J. Systematic review of information and support interventions for caregivers of people with dementia. BMC Geriatrics. 2007; 7:18. [PubMed: 17662119]

Van Mierlo LD, Meiland FJ, Van der Roest HG, Droes RM. Personalised caregiver support: effectiveness of psychosocial interventions in subgroups of caregivers of people with dementia. International Journal of Geriatric Psychiatry. 2012; 27:1-14. doi: http://dx.doi.org/10.1002/gps. 2694. [PubMed: 21520288]

Wethington, E., Burgio, LD. Translational research on caregiving: missing links in the translation process. In: Gaugler, JE., Kane, RL., editors. Family Caregiving in the New Normal. San Diego, CA: Elsevier, Inc; 2015. p. 193-210.

Zarit, SH. Empirically supported treatment for family caregivers. In: Qualls, SH., Zarit, SH., editors. Aging Families and Caregiving. Hoboken, NJ: John Wiley \& Sons, Inc; 2009. p. 131-154. 


\begin{tabular}{|c|c|c|c|c|c|c|c|}
\hline & Psychoeducational & \begin{tabular}{|c|} 
Case \\
management/counseling
\end{tabular} & $\begin{array}{l}\text { Support } \\
\text { Groups }\end{array}$ & Respite & $\begin{array}{l}\text { Training } \\
\text { of the } \\
\text { Person } \\
\text { with } \\
\text { Dementia }\end{array}$ & Psychotherapy & $\begin{array}{l}\text { Multi- } \\
\text { Component }\end{array}$ \\
\hline $\begin{array}{l}\text { 1. Initial } \\
\text { caregiver case }\end{array}$ & & & & & & & \\
\hline $\begin{array}{l}\text { 2. Care recipient } \\
\text { does not live with } \\
\text { caregiver }\end{array}$ & & & & & & & \\
\hline $\begin{array}{l}\text { 3. Care recipient } \\
\text { has a dementia } \\
\text { other than } \\
\text { Altheimer's } \\
\text { disease }\end{array}$ & & & & & & & \\
\hline $\begin{array}{l}\text { 4. Care recipient's } \\
\text { dementia severity } \\
\text { is middle stage }\end{array}$ & & & & & & & \\
\hline $\begin{array}{l}\text { 5. Care recipient's } \\
\text { dementia severity }\end{array}$ & & & & & & & \\
\hline $\begin{array}{l}\text { is late stage } \\
6 \text {. Caregiver is } \\
\text { daughter }\end{array}$ & & & & & & & \\
\hline $\begin{array}{l}\text { 7. Caregiver is } \\
\text { husband }\end{array}$ & & & & & & & \\
\hline $\begin{array}{l}\text { 8. Caregiver is not } \\
\text { husband, wife, or } \\
\text { daughter of the } \\
\text { care recipient }\end{array}$ & & & & & & & \\
\hline
\end{tabular}

Figure 1.

Example scenario set. Scenario 1: A caregiver is seeking assistance for the following reasons. The caregiver is DEPRESSED (felt depressed, sad, or cried in past month). The caregiver is also BURDENED (feels stress/strain when around the care recipient; feels stress when taking care of basic household responsibilities; does not feel good helping the care recipient). The caregiver is at-risk for poor SELF-CARE/HEALTHY BEHAVIORS (trouble sleeping in the past month; self-rated health is fair or poor). The caregiver also lacks SOCIAL SUPPORT (not very satisfied with help received from family or friends; is not very satisfied with the comfort, interest or concern provided by family or friends). However, the care recipient has low risk for BEHAVIOR PROBLEMS (caregiver has written information about dementia; caregiver does not have stress helping the care recipient in daily activities) and has no SAFETY PROBLEMS RELATED TO HER/HIS DEMENTIA (the caregiver has not felt like screaming at, yelling at, pushing, or hitting the care recipient in the past six months; the care recipient cannot get to dangerous objects; the care recipient is not left unsupervised; the care recipient does not wander or drive). The following are the initial characteristics of the caregiver and care recipient. The care recipient lives with the caregiver. The care recipient is diagnosed with Alzheimer's disease, but the care recipient's dementia severity is "early" stage (see http://www.alzheimers.org.uk/site/scripts/documents_info.php? documentID = 133 for examples of early, middle, or late stage dementia). Finally, the caregiver is the wife of the care recipient. Below, please rank order $(1=$ highest recommendation to 7 = lowest recommendation) the supportive service you believe would best suit the particular situation. In each subsequent row, one characteristic of the caregiver or the care recipient is changed. To show how this modification changes the type of service you would recommend, re-order or re-enter your service recommendations from 1 thru 7 . 
Table 1

Descriptive characteristics, dementia care professionals $(N=422)$

\begin{tabular}{|c|c|c|}
\hline & $\%$ & MEAN (STANDARD DEVIATION) \\
\hline \multicolumn{3}{|l|}{ Care discipline } \\
\hline Advance practice nurse & $5.7 \%$ & \\
\hline Registered nurse & $10.0 \%$ & \\
\hline Geriatric medicine & $1.4 \%$ & \\
\hline Family practice & $0.7 \%$ & \\
\hline Occupational therapy & $2.1 \%$ & \\
\hline Physical therapy & $0.2 \%$ & \\
\hline Psychology & $6.2 \%$ & \\
\hline Psychiatry & $1.2 \%$ & \\
\hline Public health & $1.2 \%$ & \\
\hline Primary care & $0.7 \%$ & \\
\hline Gerontology & $11.0 \%$ & \\
\hline Health administration & $3.6 \%$ & \\
\hline Social work & $36.2 \%$ & \\
\hline Other & $19.8 \%$ & \\
\hline \multicolumn{3}{|l|}{ Care sector } \\
\hline Non-profit organization (e.g. foundations or associations that provide services) & $43.5 \%$ & \\
\hline Community service provider (e.g. area agency on aging) & $5.3 \%$ & \\
\hline Assisted living & $1.0 \%$ & \\
\hline Home care/personal care & $2.4 \%$ & \\
\hline Home healthcare & $2.2 \%$ & \\
\hline Hospice & $0.7 \%$ & \\
\hline Hospital & $4.3 \%$ & \\
\hline Skilled nursing facility & $4.3 \%$ & \\
\hline Adult day service & $1.7 \%$ & \\
\hline Other & $34.7 \%$ & \\
\hline Years of experience in current discipline/practice area & & $16.54(10.87)$ \\
\hline Female & $87.2 \%$ & \\
\hline \multicolumn{3}{|l|}{ Highest degree } \\
\hline Doctorate (medical doctor, doctor of philosophy) & $14.1 \%$ & \\
\hline Master's degree & $31.7 \%$ & \\
\hline Bachelor's degree & $50.6 \%$ & \\
\hline Other & $4.5 \%$ & \\
\hline \multicolumn{3}{|l|}{ Race } \\
\hline White & $93.0 \%$ & \\
\hline Black or African American & $3.4 \%$ & \\
\hline Asian & $0.7 \%$ & \\
\hline American Indian or Alaska Native & $0.2 \%$ & \\
\hline Other & $2.6 \%$ & \\
\hline
\end{tabular}


$\% \quad$ MEAN (STANDARD DEVIATION)

\begin{tabular}{lcc}
\hline & $\%$ & MEAN (STANDARD DEVIATION) \\
\hline Ethnicity & $96.7 \%$ \\
Non-hispanic latino & $3.3 \%$ \\
Latino & \\
Geographic area of clients served & $44.8 \%$ \\
Urban & $50 \%$ \\
Suburban & $38.4 \%$ \\
Rural & $1.7 \%$ \\
Frontier & \\
\hline
\end{tabular}


Table 2

Elements of dementia caregiver scenarios

\begin{tabular}{|c|c|}
\hline RANDOMLY VARIED & SINGLE ELEMENTS VARIED SEPARATELY \\
\hline Caregiver depressive symptoms (caregiver is depressed: yes/no) & Care recipient lives with caregiver (Yes/No) \\
\hline Caregiver burden (caregiver is burdened: yes/no) & $\begin{array}{l}\text { Care recipient is diagnosed with Alzheimer's disease } \\
\text { (Yes/Other dementia diagnosis) }\end{array}$ \\
\hline Caregiver self-care (caregiver is at-risk for poor self-care: yes/no) & $\begin{array}{l}\text { Care recipient dementia severity/stage (Early, } \\
\text { Middle, Late) }\end{array}$ \\
\hline Caregiver social support (caregiver lacks social support: yes/no) & $\begin{array}{l}\text { Caregiver kin relationship to care recipient (Wife, } \\
\text { Daughter, Husband, Other relationship) }\end{array}$ \\
\hline \multicolumn{2}{|l|}{$\begin{array}{l}\text { Care recipient behavior problems (care recipient is at-risk for behavior problems: } \\
\text { yes/no) }\end{array}$} \\
\hline $\begin{array}{l}\text { Care recipient safety problems: (care recipient is at-risk for safety problems related to } \\
\text { her/his dementia: yes/no) }\end{array}$ & \\
\hline
\end{tabular}




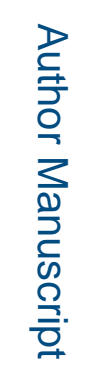

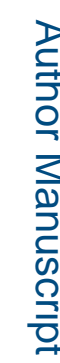

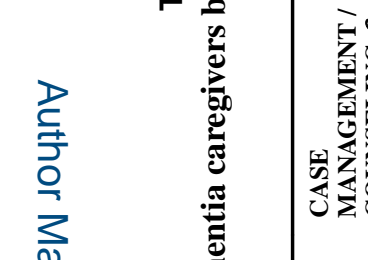

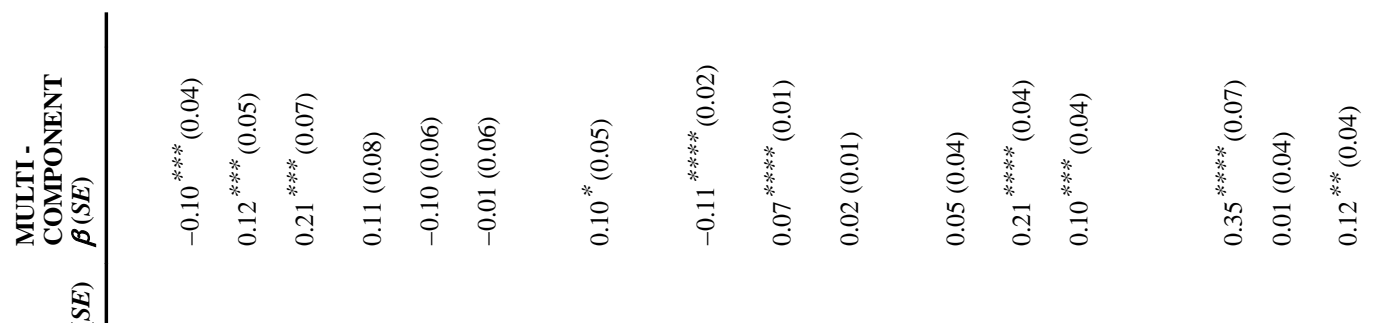

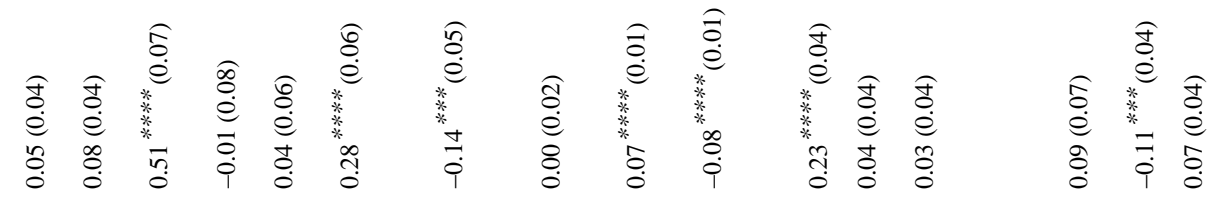

등

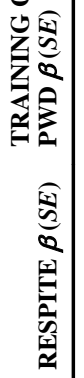

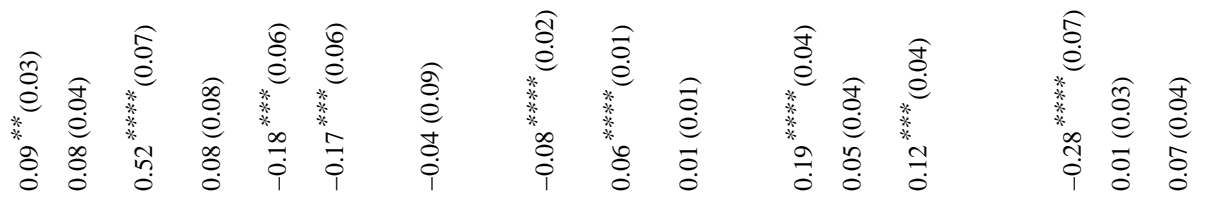

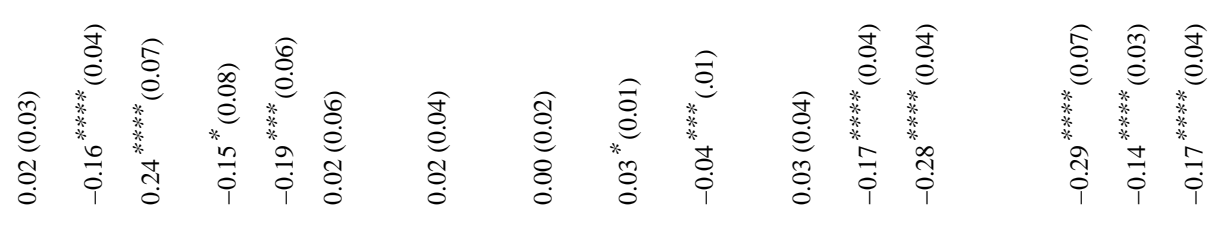

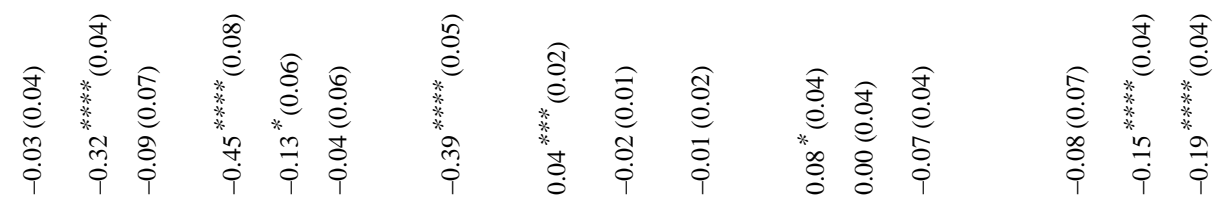

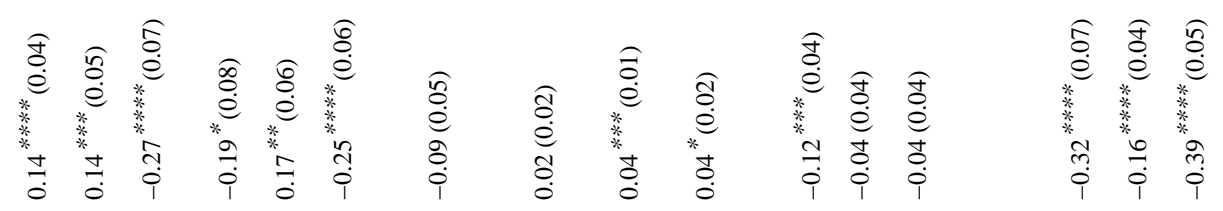

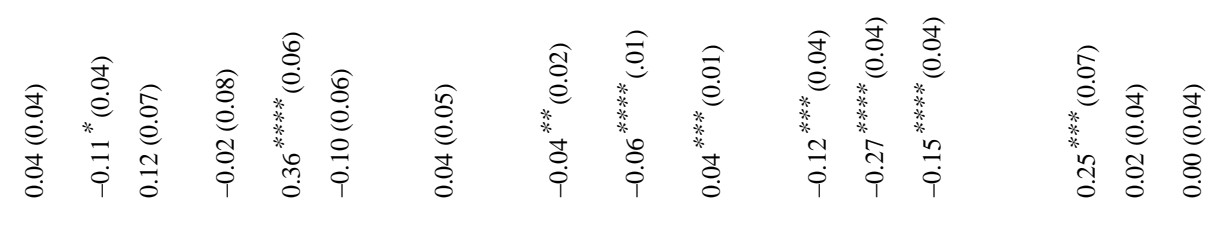

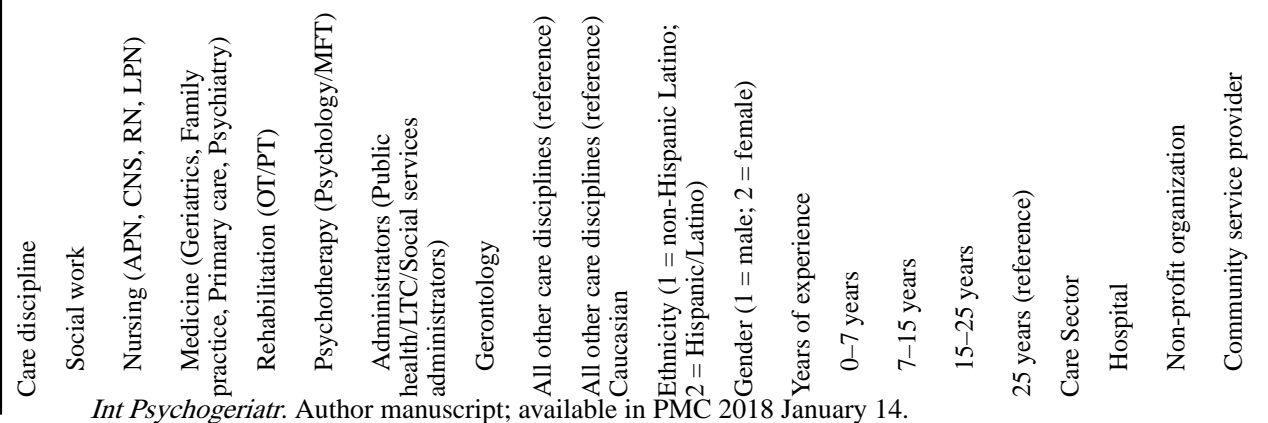




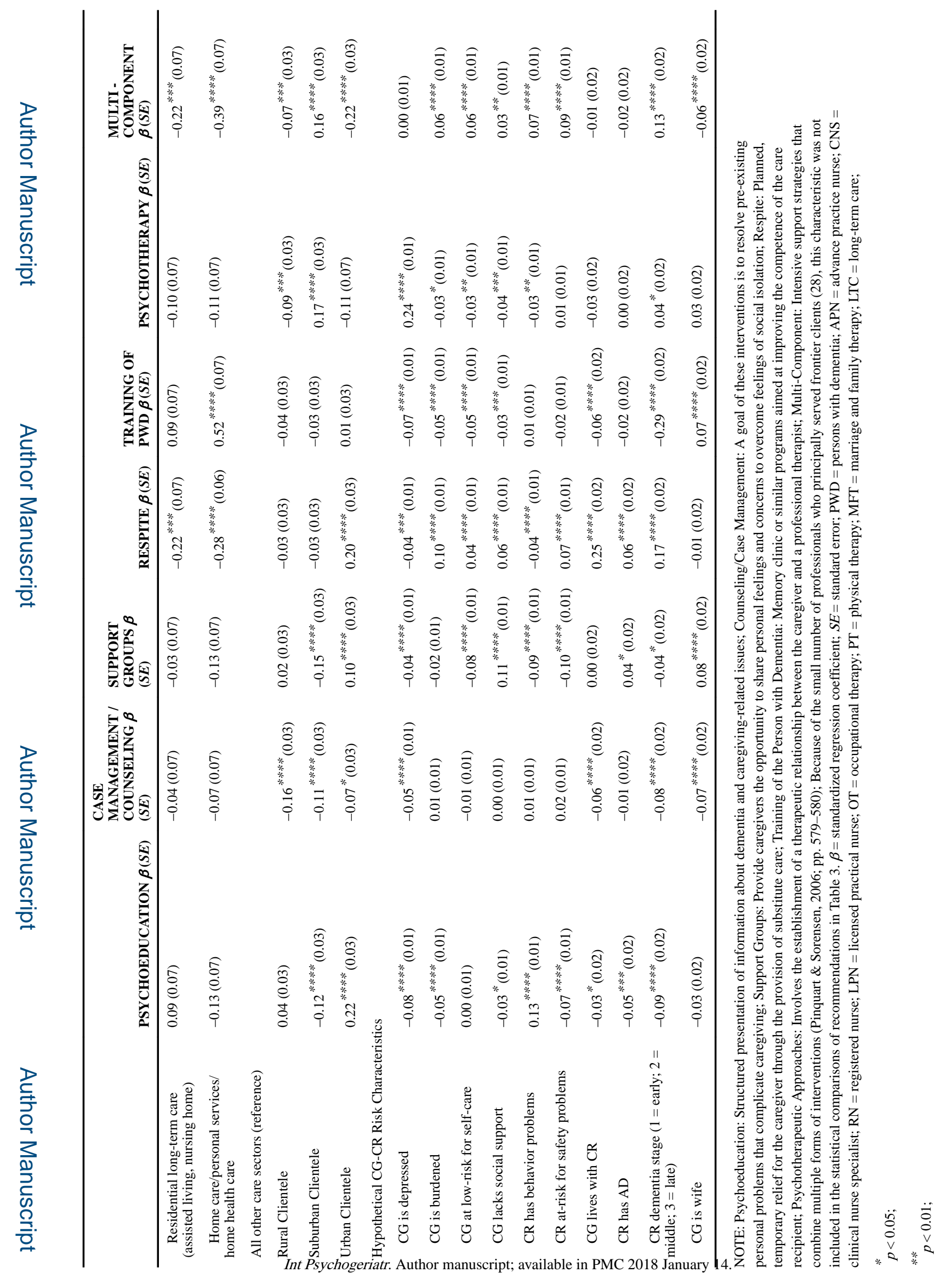




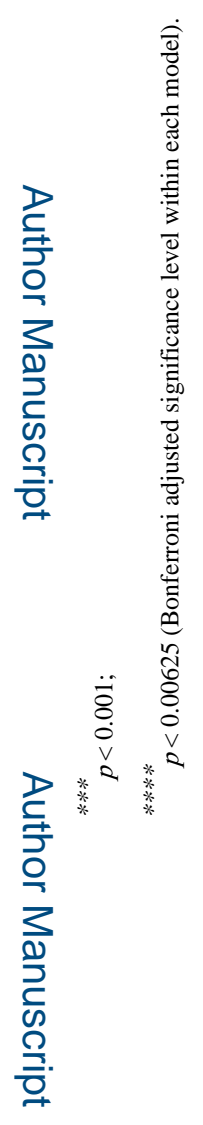

로을

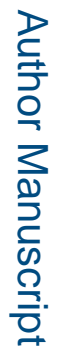

Int Psychogeriatr. Author manuscript; available in PMC 2018 January 14. 